Document downloaded from:

http://hdl.handle.net/10251/89687

This paper must be cited as:

García Moreno, E.; Pizá Fernández, R.; Quiles Cucarella, E.; Correcher Salvador, A.; Morant Anglada, FJ. (2017). Augmentation Channel Design for a Marine Current Turbine in a Floating Cogenerator. IEEE Latin America Transactions. 15(6):1068-1076. doi:10.1109/TLA.2017.7932694

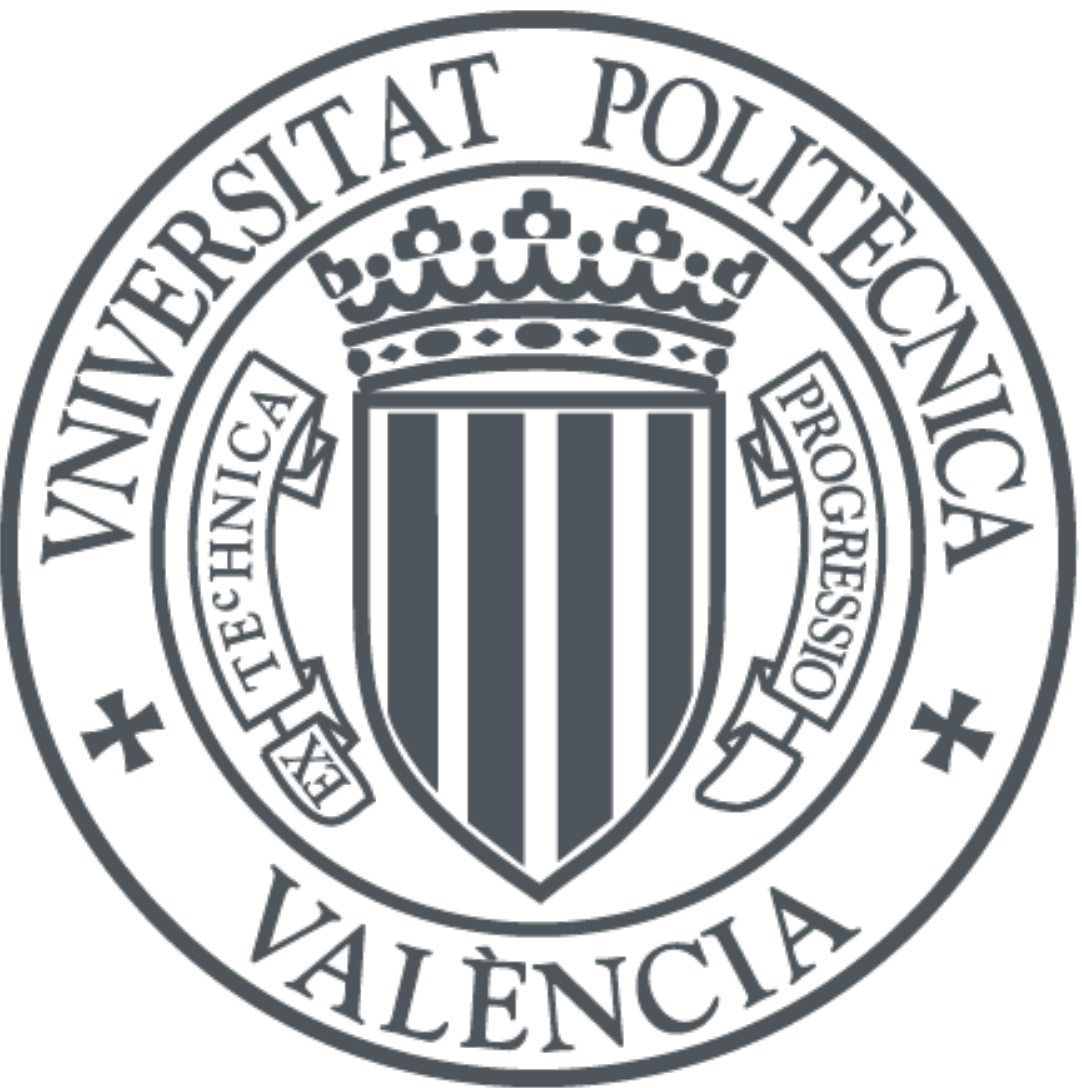

The final publication is available at

http://dx.doi.org/10.1109/TLA.2017.7932694

Copyright

Additional Information 


\title{
Augmentation Channel Design for a Marine Current Turbine in a Floating Cogenerator
}

\author{
E. García, R. Pizá, E. Quiles, A. Correcher and F. Morant
}

\begin{abstract}
In this paper we present a Hydro-Wind Kinetics Integrated Module for Renewable Energy Generation. HYWIKIM is a floating device combining wind and marine current generators for generating renewable energy. Its purpose is to exploit resources in an integrated manner using wind and current turbines in offshore plants thereby optimizing the financial investment. Our research focuses on the design and analysis of different types of augmentation channels to increase efficiency using shrouded Marine Current Turbines (MCTs) in conditions of low intensity flows.
\end{abstract}

Keywords - Floating Wind Generators; Current Turbines; Renewable Energy; Augmentation Channels; Simulation.

\section{INTRODUCCIÓN}

En estos últimos años ha habido un creciente interés hacia el desarrollo de los parques eólicos marinos (offshore) con el fin de obtener condiciones más favorables para la generación de energía renovable[1]. Las localizaciones offshore ofrecen flujos de viento de mayor intensidad, con un promedio del $30 \%$ más de los logrados en tierra. Estas condiciones permiten la instalación de turbinas eólicas más grandes, con diámetros de rotor que alcanzan e incluso superan los 120 metros y por lo tanto son capaces de generar grandes cantidades de energía. Además, estas instalaciones offshore reducen las desventajas relativas a aerogeneradores onshore en términos de contaminación visual, ruido y sus efectos sobre la avifauna.

La generación de energía marina ha aumentado recientemente con la instalación de diversos sistemas para la captación de energía de las mareas, las olas y de las corrientes marinas[2]. Específicamente las turbinas para corrientes marinas (MCTs) están en consideración por grupos de investigación y empresas. Proyectos como el SeaFlow (300KW) en Lynmouth en la costa norte de Devon o SeaGen (1.2MW) en Strangford Narrows, Irlanda del Norte, demuestran la viabilidad de este tipo de generación [3], [4].

La integración de aerogeneradores (Wind Turbines WT) y turbinas de corriente marina (Marine Current Turbines MCT) trata de optimizar la generación de energía y lograr mayores niveles de rendimiento utilizando técnicas de control multivariable, consiguiendo mayor capacidad de actuación en el sistema [5]. El proyecto Hydro-Wind Kinetics Integrated Module for Renewable Energy Generation (HYWIKIM) es un prototipo para la integración de MCTs y WT en un único sistema offshore flotante [6].

The development of this research work has been supported by the spanish CICYT DPI2010-21711-C02-01 project.

The authors are with Departamento de Ingeniería de Sistemas y Automática, Instituto Universitario de Automática e Informática Industrial, Universitat Politècnica de València, Camino de Vera s/n, 46022 Valencia (Spain). E-mail: \{egarciam, rpiza, equiles, fmorant, ancorsal\} @isa.upv.es.org
Esta integración conlleva dos ventajas: En primer lugar, podemos utilizar la estructura de flotación del aerogenerador instalando debajo de la línea de flotación dos MCTs para generar electricidad. En este sentido y con pequeñas modificaciones para la integración de las turbinas, se comparte la inversión de obra civil del aerogenerador offshore. También el sistema de canalización principal de energía es compartido por ambos sistemas.

La segunda ventaja es que los MCTs pueden utilizarse de dos maneras: como generadores de energía y también como actuadores para la estabilización del sistema. Cuando las condiciones del mar abierto amenacen la estabilidad del sistema se accionarán las MCTs con ayuda de un sistema de control multivariable inteligente. En condiciones donde es necesario priorizar la seguridad y la estabilidad, los autores proponen utilizar parte de la energía generada para aumentar el nivel de actuación del sistema de control. Esto requeriría una operación inversa de las MCTs para hacerlas trabajar como actuadores contribuyendo a la estabilización estructural del sistema flotante.

La sección II de este artículo muestra diferentes aspectos del diseño del sistema flotante. La sección III constituye el objetivo principal de este trabajo y presenta aspectos centrados en el subsistema de MCTs y el aumento de su capacidad de generación, especialmente el aumento de su eficiencia energética. Se presenta un diseño estructural basado en turbinas con canales de aumento y resultados de la simulación utilizando herramientas del software NX de Siemens combinado con ANSYS. Finalmente, la sección IV contiene un resumen y algunas conclusiones.

\section{DISEÑO DEL PROTOTIPO DE SISTEMA FLOTANTE}

Hemos implementado un sistema multifuncional basado en boyas como un banco de pruebas para la generación integrada, control, monitoreo de condiciones, diagnóstico de fallos y mantenimiento predictivo de sistemas de generación de energía renovable. También se utilizará para monitorear parámetros marinos. Este sistema integra y combina la generación de electricidad renovable offshore de las corrientes de viento y marinas (Fig. 1). El sistema contiene varios tipos de sensores para el control de estabilidad, control de condición y diagnóstico de averías y aporta datos esenciales para llevar a cabo estudios de viabilidad con respecto a sistemas de generación renovable offshore. Los datos recogidos también servirán para validar simulaciones de estos tipos de sistemas flotantes. Para más detalles sobre el proyecto HYWIKIM referimos al lector a [6].

Aunque se han sugerido algunas ideas para sistemas integrados como en [5], en el conocimiento de los autores de 
este trabajo nunca antes un sistema flotante integrado de este tipo ha sido propuesto. Hemos denominado este tipo de sistema de doble generación Hydro-Wind Kinetics Integrated Module (HYWIKIM) para generación de energía renovable. Por debajo de la línea de flotación ha sido diseñado un sistema rotativo con dos turbinas. Este diseño permite que las turbinas puedan orientarse para enfrentar adecuadamente la corriente de entrada. También permite el paso de la corriente generada en las MCTs y los datos procedentes de los sensores de corriente mediante el uso de un conector eléctrico rotatorio.

El eje de rotación para el sistema MCT y el timón fue modelado con el paquete de software para análisis dinámico Orcina Orcaflex [7]. Los coeficientes de sustentación y arrastre correspondientes a un timón NACA 63-012 fueron introducidos en el modelo para implementar el timón. Las figuras 2 y 3 , muestran las partes correspondientes al sistema de rotación. Con esta configuración, se modelaron el cuerpo completo de la boya y el sistema interno para las turbinas de rotación. En la figura 4 podemos ver cómo la combinación de dos turbinas de corriente marinas y un timón sigue la dirección de la corriente marina media $(0,2 \mathrm{~m} / \mathrm{s})$ mediante la rotación de 0 a 360 grados. No se simularon olas ni viento.

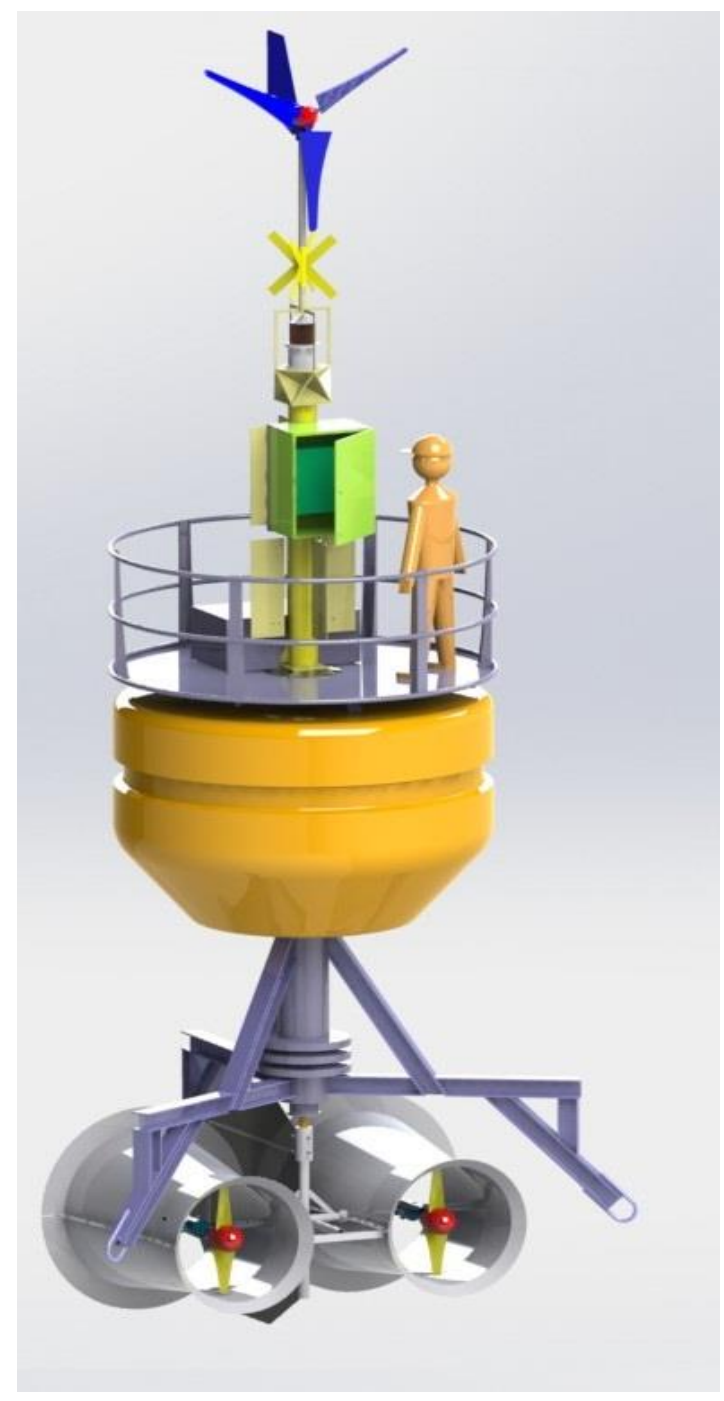

Fig. 1. Diseño CAD del prototipo HYWIKIM

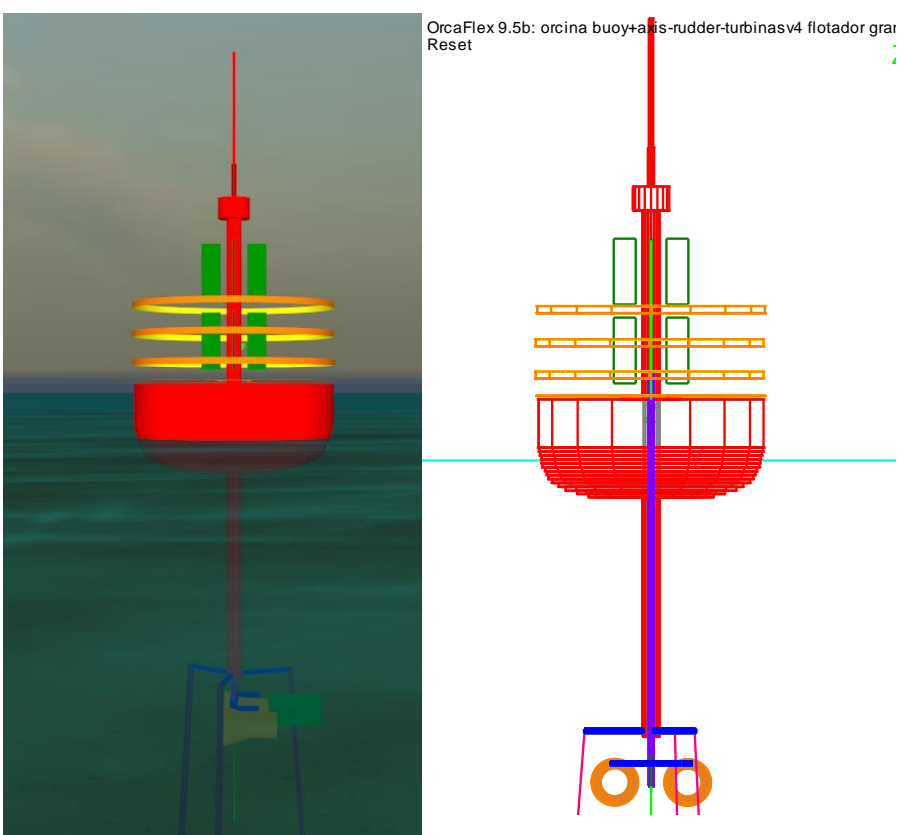

Fig. 2. Modelo desarrollado en Orcina Orcaflex.

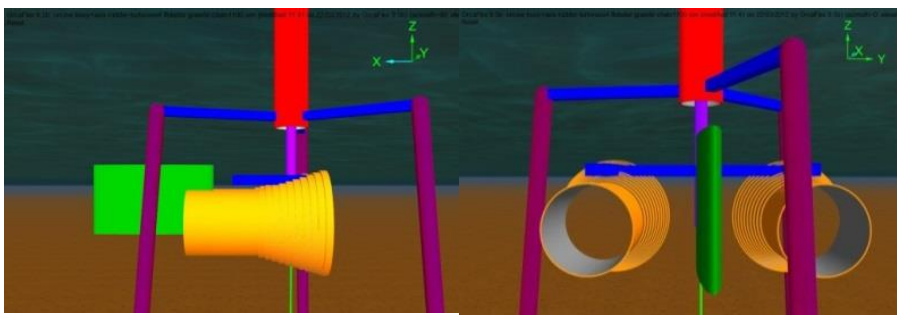

Fig. 3. Sistema rotatorio de las MCTs.

El prototipo se instalará en la costa este de España en la Comunidad Valenciana, frente a Puerto de Sagunto, a cerca de 8 millas náuticas de la costa (longitud: 39 39' 60.00", latitud: $0^{\circ} 3^{\prime} 0.00 "$ ) a una profundidad de 60 metros (Fig. 5). Se están considerando otros lugares más distantes, como el Cabo San Antonio, cerca de la localidad de Denia, para ensayos posteriores debido a sus mejores condiciones orográficas con corrientes más fuertes.

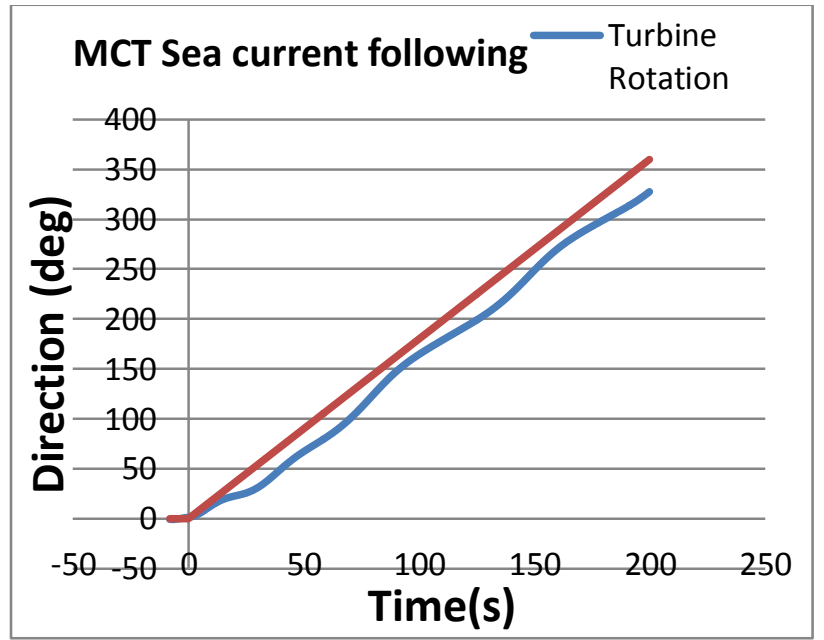

Fig. 4. Seguimiento del sistema de orientación de las MCT ante variaciones de corriente. 
Las corrientes marinas locales se pueden clasificar en tres contribuciones de energía diferentes según su frecuencia:

- Corrientes debidas a las mareas de tipo semidiurno principalmente (periodos de alrededor de 12 horas).

- Corrientes inerciales producidas por golpes de viento, que, debido a la rotación de la tierra, inducen movimientos oscilatorios en la masa de agua con frecuencia de Coriolis ( $f=2 \omega \operatorname{sen} \theta$, donde $\omega$ es la velocidad angular de rotación de nuestro planeta y $\theta$ la latitud).

- Corrientes de periodo largo o subinerciales, debido principalmente a corrientes geotróficas, inducidas por gradientes de presión barotrópicos y baroclínicos [8].

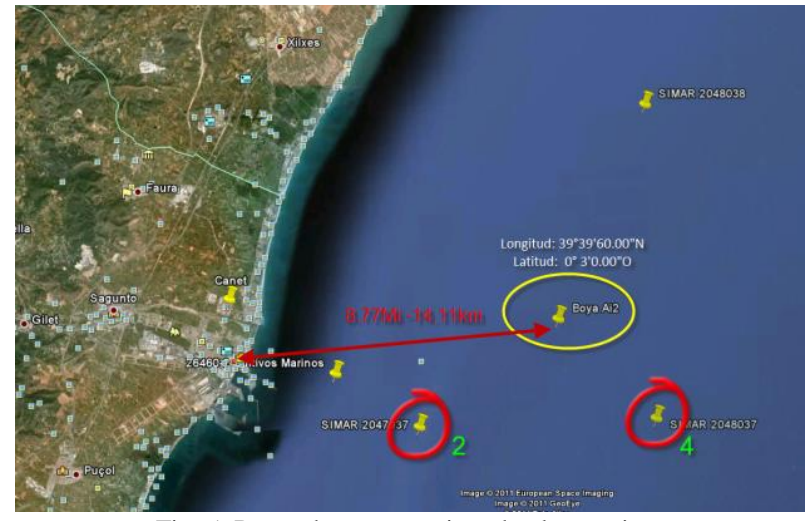

Fig. 5. Punto de amarre asignado al prototipo.

En la ubicación seleccionada los flujos de viento son considerables, pero las corrientes marinas son considerablemente más bajas que, por ejemplo, las del estrecho de Gibraltar como se aprecia en las mediciones realizadas por boyas de observación marina pertenecientes a la red de puertos del estado (Figura 6 y tabla I). Como puede verse en la tabla las corrientes medias alcanzan valores de 0.5 $\mathrm{m} / \mathrm{s}$ (con valores excepcionales de $0.86 \mathrm{~m} / \mathrm{s}$ ).

Co: Mean Currents $(\mathrm{cm} / \mathrm{s})$

Dir: Mean Direction "going to" $0=$ North $90=$ East

\begin{tabular}{lllll} 
Month & Co & Dir & Day & Hour \\
\hline January & 46.9 & 259 & 10 & 00 \\
February & 45.7 & 68 & 28 & 23 \\
March & 34.0 & 87 & 03 & 00 \\
April & 29.3 & 200 & 16 & 21 \\
May & 50.4 & 203 & 23 & 02 \\
June & 34.0 & 239 & 14 & 03 \\
July & 52.7 & 51 & 13 & 19 \\
August & 86.7 & 318 & 27 & 05 \\
September & 36.3 & 256 & 28 & 22 \\
October & 36.3 & 290 & 20 & 12 \\
November & 58.6 & 208 & 29 & 22 \\
December & 43.4 & 233 & 01 & 08 \\
\hline
\end{tabular}

Tabla I. Medidas de corriente de la boya costera Valencia II (2012).

Esta limitación y nuestro interés en extender el alcance geográfico del sistema HYWIKIM son un factor de motivación para intensificar la investigación en el aumento de la eficiencia de las turbinas de corriente marina. Con este objetivo hemos diseñado, simulado e instalado un canal de aumento en cada una de las MCTs según exponemos en la siguiente sección.

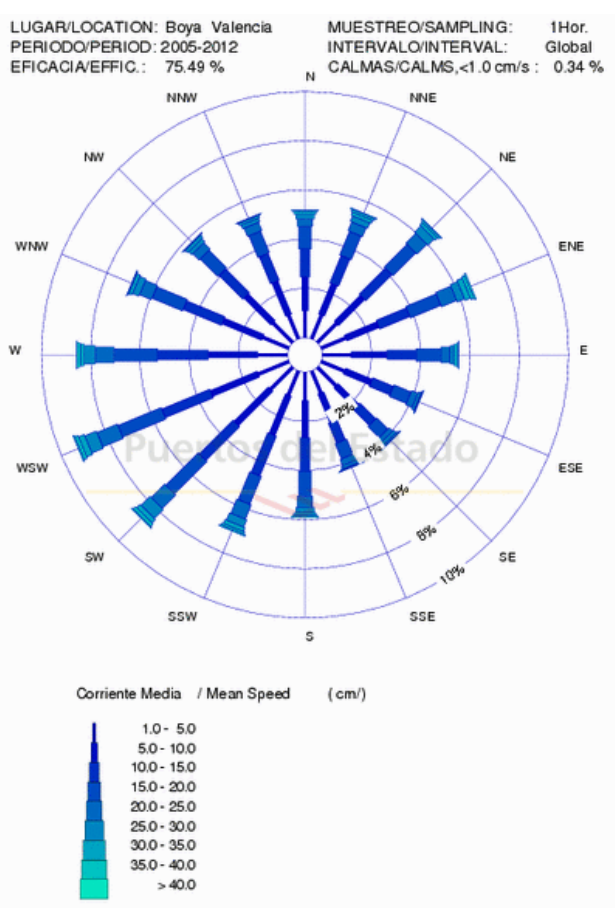

Fig. 6. Medidas de corriente del sistema de boyas costeras de Valencia [7].

\section{DISEÑO DEL CANAL DE AUMENTO}

Diversos trabajos de investigación [9] [10] [11], [12] [13] [14] [15], consideran aspectos favorables del uso de los canales de aumento (Augmentation Channels $\mathrm{ACHs}$ ) en turbinas hidrocinéticas. Una de las principales ventajas es un aumento sustancial en la tasa de flujo debido a la depresión que se origina en el interior del canal. La tasa de flujo es una variable exponencial por un factor de tres en la potencia generada por la turbina, y por lo tanto, más potencia significa un mayor retorno de inversión.

Es cierto que los canales de aumento pierden su eficacia cuando no están bien dirigidos hacia la corriente marina, pero en nuestro diseño las turbinas en condiciones normales están bien orientadas puesto que tienen un sistema rotativo controlado por un timón (Fig. 2 y 3 ).

La gama de velocidad de las corrientes de agua apta para la operación de turbinas hidrocinéticas sin ACHs se encuentra entre 1 y $3 \mathrm{~m} / \mathrm{s}$. Por debajo del límite inferior su uso no es económicamente viable, y por encima del límite superior cargas estructurales y los efectos de la cavitación pueden comprometer la integridad de las turbinas. La incorporación de los canales de aumento permite el uso de estas turbinas en muchas otras partes de la costa que de lo contrario no serían económicamente viables [16]. Otra ventaja del incremento de velocidad de flujo producida por los canales de aumento es que permite la eliminación de las etapas de baja velocidad de la caja de engranajes. Puesto que estas etapas son las partes más caras de la caja de engranajes hay una reducción directa de tamaño y costes en la máquina [17].

Aunque la aplicación de canales de aumento no ha tenido éxito con aerogeneradores de diámetro de rotor grande debido a los desafíos prácticos tales como la colocación de la cabeza de la torre, orientación variable, peso y tamaño, los ACHs en turbinas marinas aparecen más convenientes debido a su colocación bajo el agua [18]. 
El rendimiento de las turbinas con $\mathrm{ACHs}$ no está restringido por el límite de Betz. Para las turbinas con ACHs el límite teórico depende de la diferencia de presión entre la entrada al conducto y la salida y del flujo volumétrico a través del conducto. Estos factores dependen de la forma del conducto y el cociente del área del conducto al área de la turbina como muestra la figura 7.

Para realizar una serie de análisis y evaluación de varios diseños de difusor, un difusor parametrizado se muestra en la figura 8. En este difusor algunos de los parámetros pueden ser cero. Por lo tanto, este modelo puede utilizarse para todos los tipos representados en la figura 7 asignando los valores numéricos correspondientes a los parámetros.
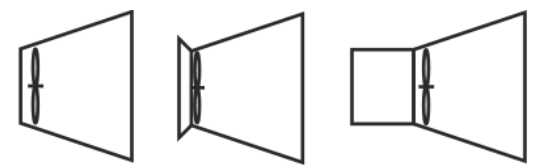

a) Simple-Diffuser

b) Nozzle-Diffuser

c) Inlet-Diffuser
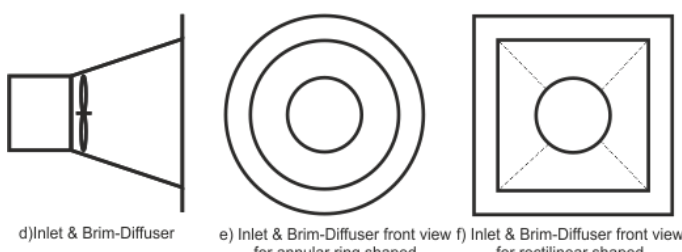

for annular ring shaped

d)Inlet \& Brim-Diffuser

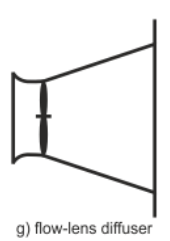

Fig. 7. Clasificación de los canales de aumento.

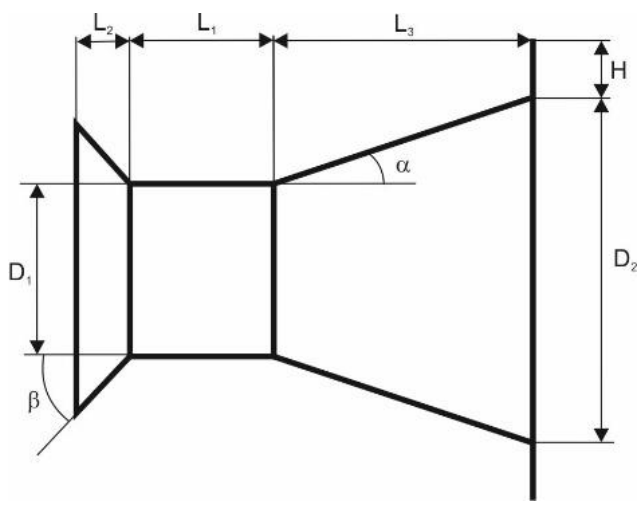

Fig. 8. Difusor tipo g (flow-lens) parametrizado.

Para aumentar la eficiencia energética de la MCTs instaladas en el prototipo HYWIKIM, se ha realizado una serie de simulaciones para encontrar el modelo óptimo. Dado que los cálculos de la dinámica de fluidos computacional (CFD) requieren altos recursos de la CPU, se ha utilizado un enfoque diferente para economizar el tiempo de cómputo. Se estudia un caso inicial de difusor vacío para determinar la geometría más adecuada para el canal de aumento. Este diseño es validado por un modelo de fuente de momento angular para simular el consumo de energía de la turbina y luego se realiza una última simulación con la geometría de la turbina completa.

La dinámica de fluidos computacional es una rama de la mecánica de fluidos que utiliza métodos numéricos y algoritmos para resolver y analizar problemas que involucran flujos de fluidos. Hay varios paquetes de software comercial disponible para simulaciones CFD de volumen finito. En esta investigación se realizaron las simulaciones numéricas con ANSYS CFX usando el método de cálculo Near-wall [19].

\section{Caso de difusor vacío}

Los estudios iniciales se hacen con un modelo de difusor vacío, es decir, sin considerar la turbina instalada en el interior del difusor.

Los modelos utilizados en las simulaciones corresponden a la figura 7 tipos a, b, c, d y g. Las velocidades de entrada para todos los ensayos se han establecido en un valor de $0.3 \mathrm{~m} / \mathrm{s}$, teniendo en cuenta las características observadas en la ubicación inicial de la costa de Valencia (tabla I).

Nuestros primeros estudios se enfocaron en los canales de aumento tipo $\mathrm{C}$ haciendo variaciones de los parámetros geométricos $\mathrm{L}$ y $\alpha$. Los resultados de estas simulaciones iniciales se presentan en la tabla II. El término eficiencia se utiliza para referirse a la expresión:

$$
\text { Eficiencia }=\frac{\widehat{V}}{V_{\text {current }}}
$$

donde $V_{\text {current }}$ es la velocidad de la corriente y $\widehat{V}$ es la velocidad obtenida en el canal de aumento.

\begin{tabular}{c|cccc}
\hline Case & $L_{3}(\mathrm{~mm})$ & $\alpha\left({ }^{\circ}\right)$ & Velocity $(\mathrm{mm} / \mathrm{s})$ & Efficiency \\
\hline 1 & 500 & 35 & 513.79 & 1.712 \\
2 & 400 & 35 & 498.75 & 1.662 \\
3 & 200 & 35 & 471.19 & 1.570 \\
4 & 300 & 60 & 443.12 & 1.477 \\
5 & 300 & 50 & 459.55 & 1.531 \\
6 & 300 & 40 & 473.3 & 1.577 \\
7 & 300 & 30 & 488.49 & 1.628 \\
8 & 300 & 35 & 480.34 & 1.601 \\
9 & 300 & 25 & 483.49 & 1.611 \\
\hline \multicolumn{5}{r}{ Tabla II. Mejores resultados de simulación del canal de aumento tipo C. }
\end{tabular}

Estos resultados no son suficientemente satisfactorios para cumplir los requisitos de nuestro prototipo y estudiamos geometrías más complejas. Se realizaron simulaciones basadas en los resultados anteriores añadiendo el parámetro geométrico $\beta$ asociado con la boquilla de entrada al difusor del canal de aumento tipo G (Fig. 8). Se realizó un estudio paramétrico que se refleja en la tabla III. El software ANSYS permite este tipo de estudio paramétrico que se realizará con el fin de establecer la dependencia geométrica de los diferentes parámetros y seleccionar rangos y restricciones.

\begin{tabular}{l|lrrrrrr}
\hline CASE & $\beta\left(^{\circ}\right)$ & $L_{1}$ & $H$ & $L_{3}$ & $\alpha\left(^{o}\right)$ & $L_{2}$ & $\begin{array}{c}\text { Effici } \\
\text { ency }\end{array}$ \\
\hline 1 & 35 & 400 & 340 & 677 & 12.5 & 100 & 1.777 \\
2 & 35 & 400 & 340 & 1579 & 12.5 & 100 & 1.897 \\
3 & 35 & 850 & 340 & 1579 & 12.5 & 100 & 1.861 \\
4 & 35 & 850 & 340 & 1579 & 12.5 & 100 & 1.862 \\
5 & 35 & 850 & 340 & 1306 & 15 & 100 & 1.738 \\
6 & 35 & 850 & 340 & 962 & 20 & 100 & 1.593 \\
\hline
\end{tabular}




\begin{tabular}{l|lllllll}
\hline 7 & 35 & 850 & 340 & 500 & 35 & 100 & 1.442 \\
8 & 35 & 850 & 340 & 294 & 50 & 100 & 1.405 \\
9 & 35 & 850 & 170 & 1579 & 12.5 & 100 & 1.86 \\
10 & 195 & 850 & 340 & 1579 & 12.5 & 100 & 1.776 \\
11 & 200 & 850 & 340 & 1579 & 12.5 & 100 & 1.811 \\
12 & 50 & 850 & 340 & 1579 & 12.5 & 100 & 1.687 \\
13 & 0 & 850 & 340 & 1579 & 12.5 & 100 & 1.656 \\
14 & 35 & 850 & 340 & 1579 & 12.5 & 250 & 1.858 \\
15 & 0 & 850 & 50 & 1579 & 12.5 & 25 & 1.705 \\
16 & 35 & 850 & 340 & 2659 & 7.5 & 250 & 2.347 \\
17 & 35 & 850 & 540 & 1579 & 12.5 & 250 & 1.757 \\
18 & 35 & 850 & 340 & 677 & 12.5 & 100 & 1.809 \\
19 & 35 & 850 & 340 & 677 & 12.5 & 25 & 1.686 \\
20 & 35 & 850 & 170 & 677 & 12.5 & 100 & 1.736 \\
21 & 35 & 850 & 50 & 677 & 12.5 & 100 & 1.746 \\
22 & 35 & 400 & 170 & 2481 & 12.5 & 100 & 1.91 \\
23 & 35 & 400 & 170 & 1139 & 7.5 & 100 & 2.042 \\
24 & 35 & 400 & 170 & 1715 & 5 & 100 & 2.088 \\
25 & 35 & 400 & 10 & 1715 & 5 & 100 & 1.743 \\
26 & 35 & 400 & 340 & 1715 & 5 & 100 & 2.12 \\
27 & 35 & 850 & 340 & 2659 & 7.5 & 100 & 2.362 \\
28 & 35 & 850 & 340 & 4001 & 5 & 100 & 2.55 \\
29 & 35 & 850 & 340 & 1139 & 7.5 & 100 & 2.02 \\
30 & 35 & 400 & 340 & 677 & 7.5 & 100 & 2.324 \\
\hline \multicolumn{5}{r}{ Tabla III. Mejores resultados de simulación del canal de aumento }
\end{tabular}

Puesto que el modelo es axisimétrico, una porción de $6^{\circ}$ de todo el sistema se utiliza para la simulación, permitiendo una malla más refinada sin extender el tiempo de cálculo. Para construir el modelo se genera un cilindro de agua y dentro de este volumen se crea el $\mathrm{ACH}$. Las dimensiones del cilindro deben ser suficientes para que las paredes no sean afectadas por la turbulencia en el flujo causado por el $\mathrm{ACH}$. El volumen del $\mathrm{ACH}$ se resta del volumen de agua para obtener el espacio físico ocupado por el agua en la prueba. Según las condiciones de límite, un flujo de entrada se aplica a través de una base del cilindro y el flujo de salida por el otro extremo. Se aplica una malla sobre este volumen para obtener los nodos que definen los elementos finitos en la estructura.

El mejor rendimiento se logra en el caso 28 con un ratio de 2.55. Para optimizar también la economía del sistema se calculan las razones de volumen y longitud de las diferentes geometrías estudiadas. Se incluyen en la tabla con el fin de evaluar la mejor solución para lograr una relación de por lo menos 2 y que utilice menos volumen y menos materiales (Tabla IV). El caso 23 es el sistema más pequeño que cumple el requisito.

\begin{tabular}{l|crrrrrr}
\hline Case & Ratio & $\begin{array}{c}L_{3} \\
(\mathrm{~m})\end{array}$ & $\begin{array}{c}\mathrm{D}_{1} \\
(\mathrm{~m})\end{array}$ & $\begin{array}{c}\mathrm{D}_{2} \\
(\mathrm{~m})\end{array}$ & $\begin{array}{c}\text { Vol } \\
\text { ume }\end{array}$ & $\begin{array}{c}\text { Ratio/ } \\
\text { Length }\end{array}$ & $\begin{array}{c}\text { Ratio/ } \\
\text { Volume }\end{array}$ \\
\hline 16 & 2.34 & 3.75 & 1.25 & 2.28 & 6.66 & 0.624 & 0.352 \\
23 & 2.04 & 1.63 & 1.04 & 1.54 & 1.48 & 1.246 & 1.376 \\
24 & 2.08 & 2.21 & 1.04 & 1.54 & 2.00 & 0.943 & 1.041 \\
26 & 2.12 & 2.21 & 1.04 & 1.88 & 2.68 & 0.957 & 0.791 \\
27 & 2.36 & 3.60 & 1.04 & 2.28 & 5.93 & 0.655 & 0.398 \\
28 & 2.55 & 4.95 & 1.04 & 2.28 & 8.14 & 0.515 & 0.313 \\
29 & 2.02 & 2.08 & 1.04 & 1.88 & 2.52 & 0.967 & 0.799 \\
30 & 2.32 & 2.39 & 1.04 & 2.08 & 3.40 & 0.969 & 0.683 \\
\hline
\end{tabular}

parámetros geométricos del difusor tipo $\mathrm{g}$ (flow-lens).

\section{Modelo de fuente de momento}

En [20] y [21] se estudia el uso de difusores para mejorar el rendimiento y la viabilidad de hidroturbinas. Para simplificar el problema, la turbina se modela como una región de fuente de momento, es decir, no se modeliza la turbina y en la simulación se introduce un momento de inercia adicional.

El rotor de la turbina representa un desafío para el modelamiento numérico debido a la complejidad de su geometría y al movimiento de rotación. Esto es complicado, aún más si de un problema de optimización se trata, puesto que se requieren numerosas simulaciones en los cálculos. La inclusión de un rotor de turbina extendería el tiempo de cómputo considerablemente en un estudio de optimización. Puesto que una resolución completa del flujo a través del rotor no es necesaria para la optimización de los canales de aumento, es posible simplificar el sistema con el modelo de la fuente de momento.

La turbina afecta a la solución de flujo final introduciendo un efecto no lineal. La perturbación de la presión de la turbina depende de la velocidad que a su vez depende de la presión de salida del difusor. El modelado de la fuente de momento es válido para simular este cambio de presión.

Con este método se define un subdominio dentro del difusor donde debería estar la turbina. En este subdominio se extrae parte de la energía del líquido del sistema en la forma en que lo haría la turbina mediante la introducción de una fuente de momento en la región cilíndrica que contiene el rotor la turbina. En [22] se ofrece un estudio detallado de la teoría de fuente de momento que es una estrategia común para modelado en aerogeneradores. En [23] el rotor se modela como una matriz de puntos discretos de fuente de momento que han sido derivados empíricamente.

El modelo de fuente de momento se logra mediante la eliminación de la geometría de la turbina, dejando un paso abierto. En su lugar, se aplica una fuente de momento uniformemente a través de una región de la turbina de longitud $l$, haciendo que el fluido pierda la energía que la turbina extraería normalmente [21]. La fuente de momento viene dada por:

$$
\frac{\partial s}{\partial t}=C_{s} \frac{\rho u^{2}}{2 l}
$$

Donde $s$ es el momento por unidad de volumen de fluido, $\rho$ es la densidad, $u$ es la velocidad local, $l$ es la longitud a lo largo de la línea de corriente de la región de la turbina y $C_{s}$ es el factor de fuente de momento, una constante que se relaciona con el coeficiente de empuje de la turbina.

$$
C_{s}=C_{T} \frac{l A\left(u_{\infty}\right)^{2}}{\int u^{2} d V}
$$

Donde $A$ es el área de turbina, $V$ el volumen de la turbina y $\mathrm{u}_{\infty}$ la velocidad de la corriente. Si las variaciones de velocidad dentro de la región de la turbina se supone que son pequeñas, la expresión se reduce a:

$$
C_{s}=C_{T}\left(\frac{u_{\infty}}{u_{t}}\right)^{2}
$$

La potencia eficaz de la turbina y el arrastre total están dados por:

$$
W=C_{T} \frac{\rho u_{t} u_{\infty} A}{2}
$$




$$
F_{\text {total }}=F_{\text {shroud }}+F_{\text {turbine }}=F_{\text {shroud }}+\frac{W}{u_{t}}
$$

donde $F_{\text {shroud }}$ es el arrastre en el conjunto de la cubierta, una cantidad que puede extraerse de los resultados numéricos mediante la integración de la distribución de presión en el conjunto de la cubierta.

Los resultados de esta optimización se reflejan en la tabla $\mathrm{V}$ $\mathrm{y}$ algunos resultados gráficos se muestran en las figuras 9 y 10. El subdominio se refleja en esta figura con una longitud de $100 \mathrm{~mm}$. Se observan algunas diferencias entre el caso vacío y esta simulación. La caída de presión es menos pronunciada causando un cambio más suave en el flujo de corriente.

Se ha realizado varias investigaciones sobre cómo el ángulo del difusor afecta el rendimiento de las turbinas de corriente marinas con estudios que detallan mejoras hasta ángulos de $20^{\circ}$, pero más allá de ese valor el aumento es insignificante. Estos resultados se confirman en [21]. El estudio también considera la proporción de área de entrada y salida y su efecto en el coeficiente de arrastre, concluyendo que una relación de área de 1.56 es un compromiso satisfactorio entre el aumento de la velocidad y los efectos de arrastre en una configuración de difusor rectilíneo.

Comparando con los resultados del caso de difusor vacío, es notable la disminución en la eficiencia ya que no se modela el efecto de la turbina en el caso del difusor vacío.

\begin{tabular}{l|lrrrrrr}
\hline CASE & $\beta\left(^{\circ}\right)$ & $L_{1}$ & $H$ & $L_{3}$ & $\alpha\left(^{\circ}\right)$ & $L_{2}$ & $\begin{array}{c}\text { Effici } \\
\text { ency }\end{array}$ \\
\hline 1 & 35 & 400 & 170 & 1139 & 7.5 & 100 & 1.887 \\
2 & 30 & 400 & 170 & 1139 & 7.5 & 100 & 1.885 \\
3 & 40 & 400 & 170 & 1139 & 7.5 & 100 & 1.864 \\
4 & 35 & 400 & 170 & 1139 & 8.75 & 100 & 1.745 \\
5 & 30 & 400 & 170 & 1139 & 8.75 & 100 & 1.744 \\
6 & 40 & 400 & 170 & 1139 & 8.75 & 100 & 1.736 \\
7 & 35 & 400 & 170 & 1139 & 10 & 100 & 1.728 \\
8 & 30 & 400 & 170 & 1139 & 10 & 100 & 1.699 \\
9 & 40 & 400 & 170 & 1139 & 10 & 100 & 1.701 \\
\hline \multicolumn{7}{r}{ Tabla V. Resultados de simulación para el modelo de fuente de momento. }
\end{tabular}

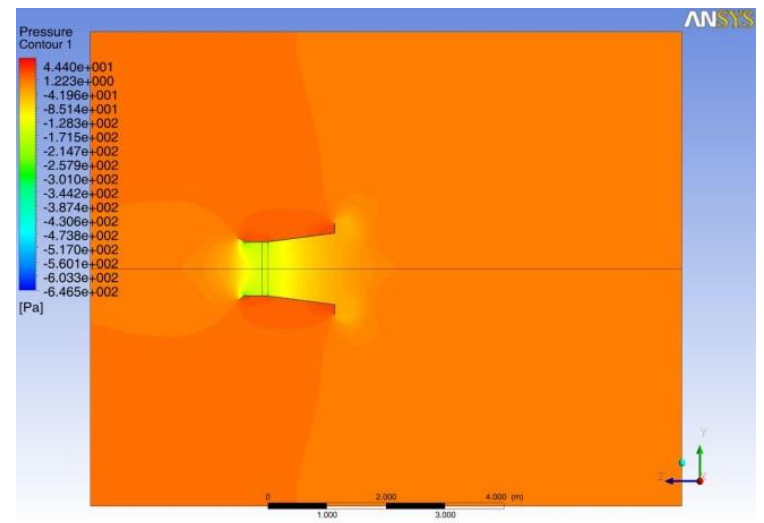

Fig.9. Simulación del canal de aumento tipo g. Presión reflejada en el plano de simetría. Modelo de fuente de momento.

Además se evaluó otra alternativa: Igual que en [24] donde una superficie de sustentación NACA 0016 es evaluada para el diseño de un difusor, se utilizó un diseño de superficie de sustentación de GOE81 para evaluar esta posibilidad. El difusor obtenido mediante este perfil se muestra en la Fig. 11. Después de la simulación (Fig. 12), con las mismas condiciones, los resultados no mejoran los obtenidos con los diseños más convencionales utilizados en las simulaciones anteriores.

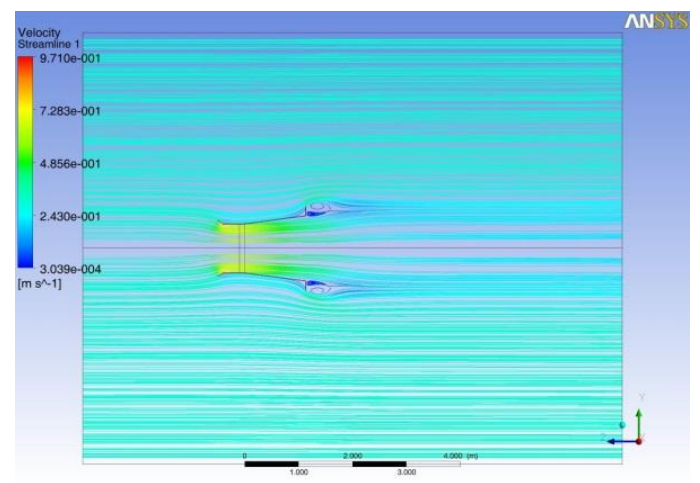

Fig. 10. Simulación del canal de aumento. Líneas de corriente reflejadas en el plano de simetría. Modelo de fuente de momento.
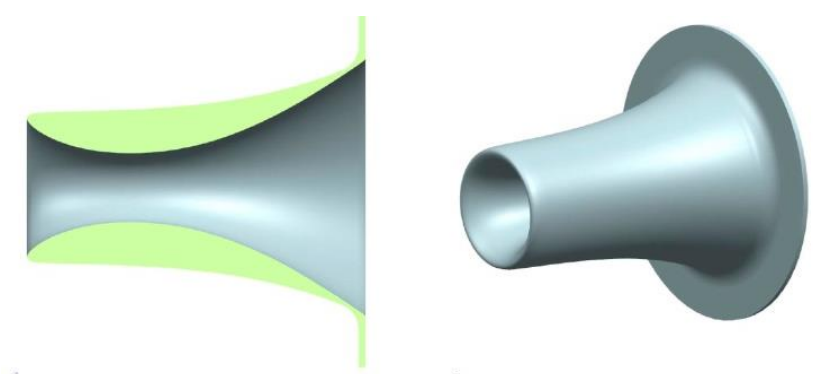

Fig. 11. Difusor obtenido con un diseño GOE81.

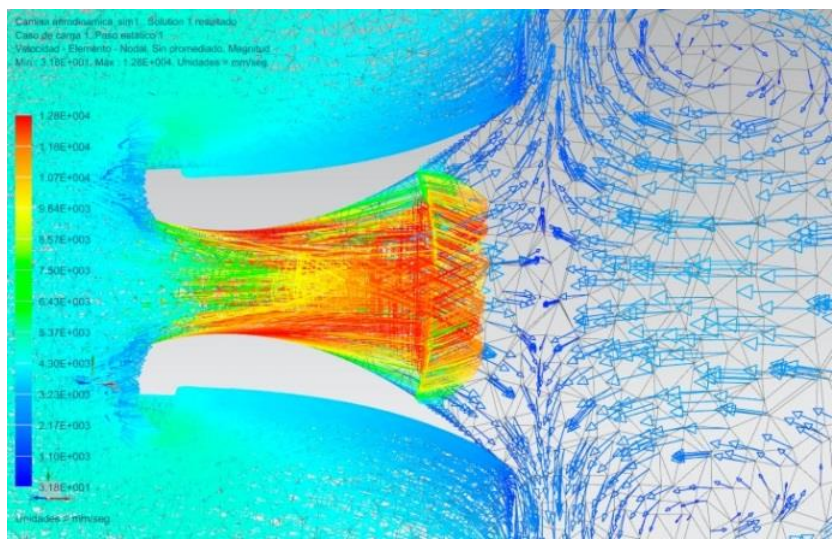

Fig. 12. Resultados de simulación obtenidos con un modelo GOE81.

\section{Modelo de rotor congelado}

Se realizó una simulación con el rotor congelado, es decir, la hélice de la turbina bloqueada, para obtener una imagen detallada de la distribución de las líneas de corriente en la turbina y de la presión en las paredes del sistema. El modelo de rotor congelado se utiliza habitualmente en simulaciones de aerogeneradores. Este modelo reduce el tiempo de simulación y produce una solución aproximada. La región de rotación no gira durante la simulación. El momento de rotación y términos asociados son asignados al flujo en su lugar. Una porción alrededor de las palas se configura como un subdominio separado que se define como de rotación, mientras que el contorno está definido como estático. Se define una interfaz entre el rotor y el estator.

En este caso la velocidad de rotación se considera $70 \mathrm{rpm}$ que es la velocidad esperada a la entrada de la turbina. El 
resultado se presenta en la figura 13. La velocidad media obtenida en el canal de aumento valida los resultados del modelo de la fuente de momento. La turbulencia causada por el rotor junto con el efecto del canal de aumento generalmente produce vibraciones en el sistema. Este efecto es importante en los sistemas donde las corrientes son altas. Sin embargo, en el sistema que estamos considerando, ya que las corrientes son débiles, este efecto es despreciable.

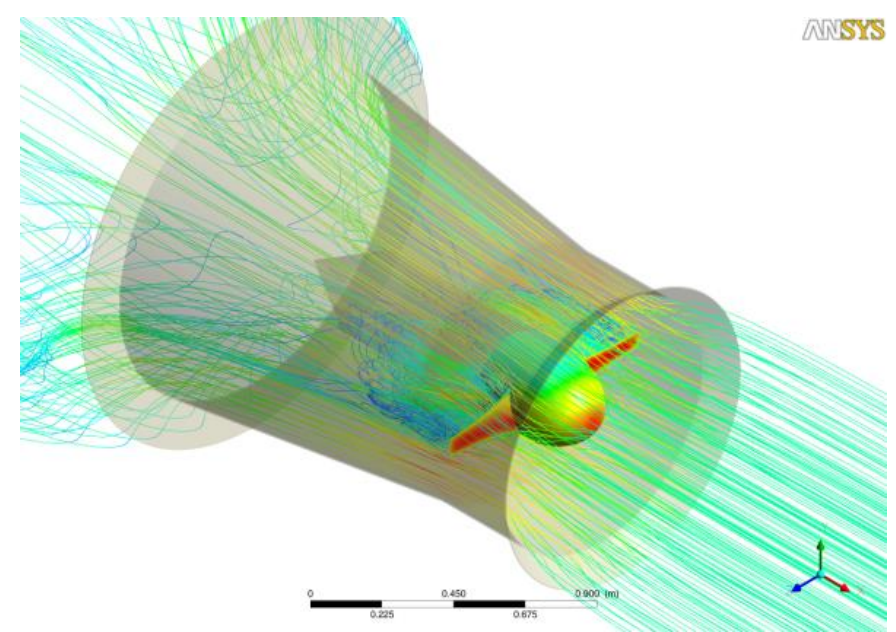

Fig. 13. Simulación de las líneas de corriente en el modelo de rotor congelado.

\section{CONCLUSIONES}

El concepto HYWIKIM integra cogeneración de aerogeneradores y turbinas de corriente marina utilizando una estructura de flotación individual. Este prototipo está destinado a ser utilizado para adquisición de datos e identificación de parámetros de recursos renovables en el mar (flujos de viento y corrientes marinas), así como para análisis de estabilidad de la estructura, estrategias de control inteligente y diagnóstico de fallos.

HYWIKIM es un sistema complejo con seis grados de libertad, que requiere estrategias de control multivariable inteligente, permitiendo el control integrado de los dispositivos de generación instalados. Sin embargo, en condiciones de máxima inestabilidad debido a causas meteorológicas, las estrategias de control deben asegurar la estabilidad del sistema mediante la operación reversible de las turbinas de corriente marina.

Se ha realizado el análisis y diseño de los canales de aumento para aumentar la eficiencia de MCTs en el ámbito del proyecto HYWIKIM. Con el canal de aumento tipo $\mathrm{C}$ el aumento de la eficiencia fue de 1.712. Para el tipo $G$ se consiguió una eficiencia máxima de 2.55. Para este último modelo se realizó un análisis de variación paramétrica. De las simulaciones se concluye que la mejor opción para nuestro prototipo está basada en el uso del tipo $G$ de canal de aumento. Se utilizó un modelo de fuente de momento para validar el sistema teniendo en cuenta el efecto de la turbina y se realizó una simulación modelando la geometría de la hoja entera con el fin de validar el diseño final.
Los resultados de la simulación se han utilizado para implementar un canal de aumento reconfigurable en nuestro prototipo (Fig. 14). La segunda fase experimental de este trabajo tendrá en cuenta la influencia de diferentes factores en la eficiencia de la turbina directamente a partir de nuestro prototipo (Fig. 15) con el objetivo de adaptar el diseño a las corrientes de agua existentes en nuestra área de interés en el mar Mediterráneo.

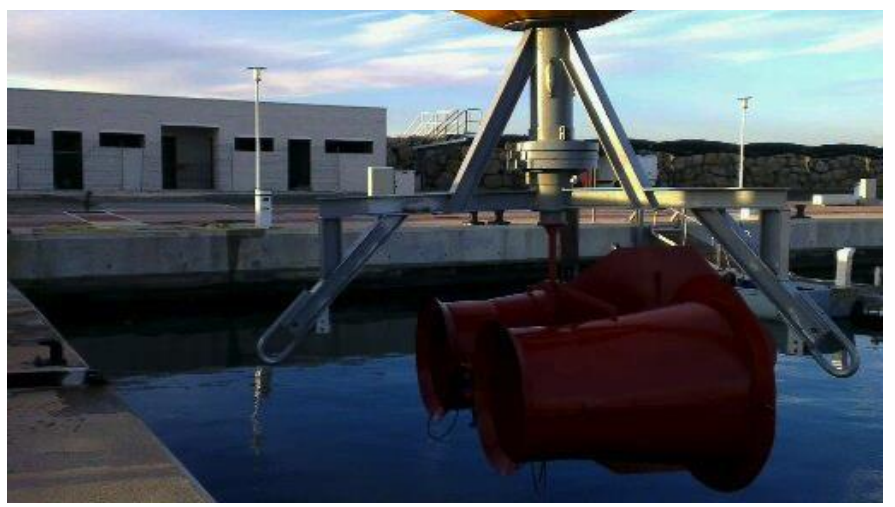

Fig. 14. Implementación de los canales de aumento en el prototipo.

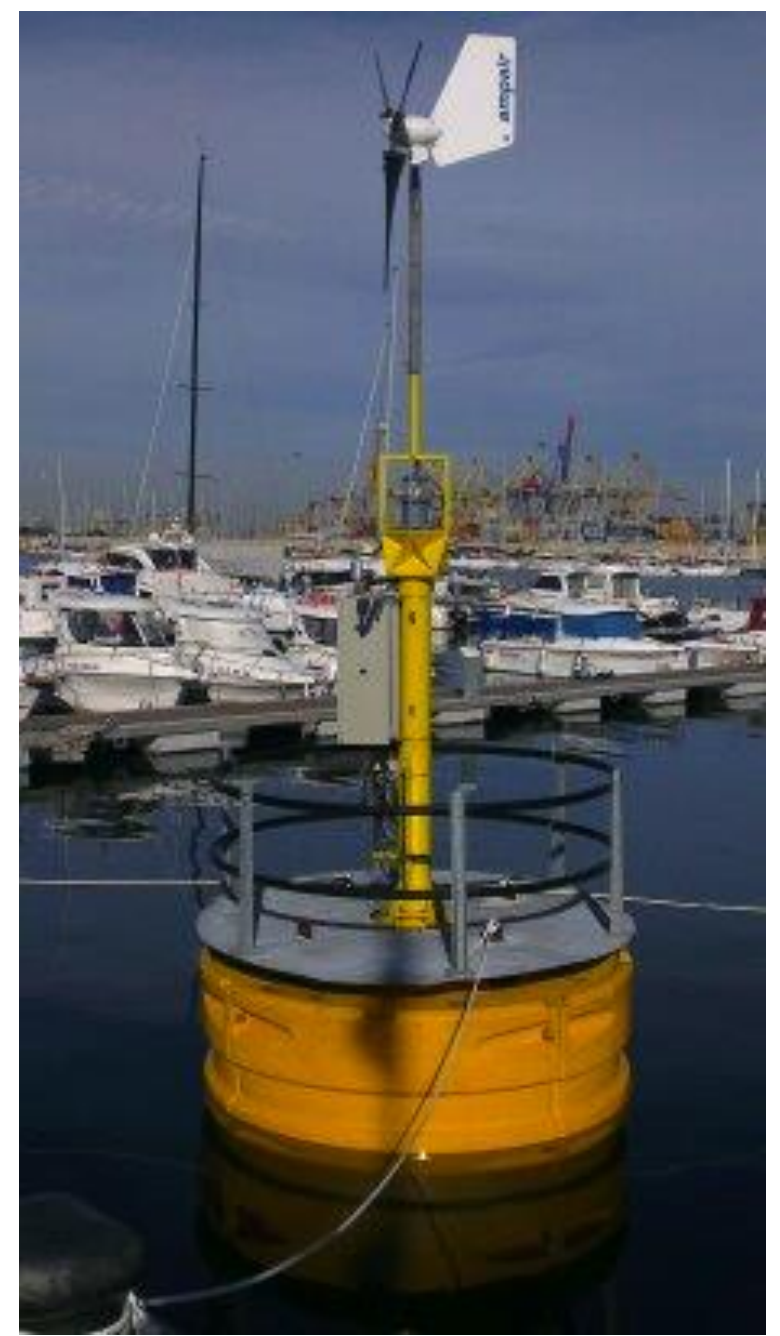

Fig. 15. Ensayos hidroestáticos e hidrodinámicos en el puerto del Real Club Náutico de Valencia. 


\section{AGRADECIMIENTOS}

Especialmente destacable es la colaboración del Real Club Náutico de Valencia y de la autoridad portuaria de Valencia (Puerto de Valencia). Los autores también agradecen a los siguientes estudiantes de Erasmus de la Universidad de Strathclyde por su colaboración en simulaciones CFD: Ewan Baillie, Alan Carrie, Douglas Currie y Ewan Keddie.

\section{REFERENCIAS}

[1] Queiroz Oliveira, O.D.; Araujo, A.M.; Ramos Medeiros, A.L.; Pereira Silva, H.; Rohatgi, J.S.; Asibor, A.I. A Preliminary Approach of the Technical Feasibility of Offshore Wind Projects along the Brazilian Coast. Latin America Transactions, IEEE. Year: 2013, Volume: 11 Issue: 2. Pages: $706-712$

[2] Fischer, A.; d'Elia Machado, C.L.; Brito de Almeida, L.E.; Beluco, A. Energetic Potential and Variability of Ocean Currents on the Southern Coast of Brazil. Latin America Transactions, IEEE. Year: 2015, Volume: 13, Issue: 5. Pages: 1369 - 1375.

[3] S.E. Ben Elghali, M.E.H. Benbouzid, J.F. Charpentier. "Marine Tidal Current Electric Power Generation Technology: State of the Art and Current Status," Electric Machines \& Drives Conference, 2007. IEMDC '07. IEEE International, vol.2, no., pp.1407-1412, 3-5 May 2007.

[4] S.E. Ben Elghali, M.E.H. Benbouzid, T.Ahmed-Ali, J.F. Charpentier, F.Mekri. "High-order sliding mode control of DFIG-based marine current turbine," Industrial Electronics, 2008. IECON 2008. 34th Annual Conference of IEEE, vol., no., pp.1228-1233, 10-13 Nov. 2008.

[5] G. Caraiman, C. Nichita, V. Mînzu, B. Dakyio, C.H. Jo. ”Concept study of offshore wind and tidal hybrid conversion based on real time simulation" International Conference on Renewable Energies and Power Quality (ICREPQ'11) Las Palmas de Gran Canaria (Spain), 13th to 15th April, 2011.

[6] E. García, D. Fabuel, R. Pizá, F. Morant, A. Correcher, E. Quiles. "Hydro-Wind Kinetics Integrated Module for the Renewable Energy Generation" MTS/IEEE Oceans 2012 Yeosu, Korea 21-24 May 2012

[7] http://www.orcina.com.

[8] http://www.puertos.es.

[9] B. Kirke. (2005, Aug) Developments in ducted water current turbines. Tidal paper 16-08-03 1. Available: http://www.cyberiad.net/tide.htm.

[10] M. J. Khan; M. T. Iqbal; J. E. Quaicoe. "A Technology Review and Simulation Based Performance Analysis of River Current Turbine Systems," Electrical and Computer Engineering, 2006. CCECE '06. Canadian Conference on , vol., no., pp.2288-2293, May 2006

[11] R. L. Radkey and B. D. Hibbs. "Definition of cost effective river turbine designs," Report for U.S. Department of Energy. Pasadena, California, Tech. Rep. AV-FR-81/595 (DE82010972), Dec. 1981.

[12] http://www.lunarenergy.co.uk/

[13] http://www.hidrohelix.fr/

[14] G. Riegler. Principles of energy extraction from a free stream by means of wind turbines. Wind Engineering 7/2, 1983, p.115-126.

[15] B.L. Gilbert, and K.M. Foreman. Experiments with a diffuseraugmented model wind turbine. J. Energy Resources Technology, Trans ASME, vol.105, March 1983, p.46-53.

[16] J. King, T. Tryfonas. "Tidal Stream Power Technology-State of the Art". In Proceedings of the OCEANS 2009-EUROPE, Bremen, Germany, 11-14 May 2009; pp. 1-8.

[17] F.L. Ponta, P.M. Jacovkis. "Marine-current power generation by diffuser augmented floating hydro-turbines" Renewable Energy, Volume 33, Issue 4, April 2008, Pages 665-673. Elsevier.

[18] M.J. Khan, M.T. Iqbal, J.E. Quaicoe. "River current energy conversion systems: Progress, prospects and challenges", Renewable and Sustainable Energy Reviews. 12 (2008) 2177-2193.

[19] http://www.ansys.com

[20] D.L.F. Gaden. "An investigation of river kinetic turbines: performance enhancements, turbine modeling techniques, and an assessment of turbulence A thesis presented to the Faculty of Graduate Studies,University of Manitoba"

[21] D.L.F. Gaden, E.L. Bibeau. Renewable Energy, 2010 - Elsevier "A numerical investigation into the effect of diffusers on the performance of hydro kinetic turbines using a validated momentum source turbine model"
[22] R. Mikkelsen, J.N. Sørensen, W.Z. Shen. "Modelling and analysys of the flow field around a coned rotor". Wind Energy 2001:4:121-35

[23] C. Yihua and Y. Ziwen. "Numerical simulation of turbulent flow around helicopter ducted tail rotor". Aerospace Science and Technology 2005:9:300-5

[24] N. Mehmood, Z. Liang and J. Khan. 2012. Diffuser augmented horizontal axis tidal current turbines. Res. J. Appl. Sci. Eng. Technol., 4(18): 3522-3532. 2012

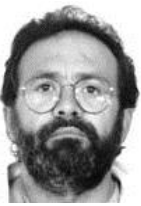

Emilio García Moreno worked in the shipyards of the Union Naval de Levante while simultaneously studying Electrical Industrial Technical Engineering at the School of Industrial Technical Engineering of the Polytechnic University of Valencia. He received a degree in February 1988. He undertook further studies in electronics in the Faculty of Physics of the University of Valencia and received a second degree in October 1995. He obtained his Ph.D. degree from the Polytechnic University of Valencia in June 2000. Since 1989 he has been with the Department of Systems Engineering and Control of the Polytechnic University of Valencia as an assistant lecturer. His research interests are in discrete-event and hybrid systems (control, failure diagnosis, and applications in factory automation and control systems).

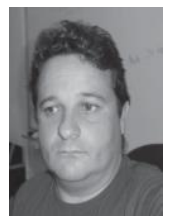

Ricardo Pizá received the B.Eng. and Ph.D. degrees in control engineering from Valencia Technical University, Valencia, Spain, in 1997 and 2003, respectively. He is currently an Assistant Professor with the Universitat Politècnica de València, Valencia. He has authored or coauthored several papers in journals and conferences, and has been involved in several research projects funded by local industries and government. His current research interests include network-based control systems, computer aided manufacturing, and robotics.

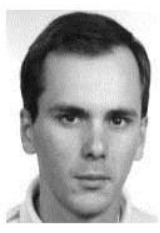

Eduardo Quiles Cucarella received an M.Sc in Electrical Engineering from the Polytechnic University of Valencia in 1993, and a Ph.D. from the same university in 1998. In 1996 he joined the Systems and Control Engineering Department of the Technical University of Valencia, where he is an associate lecturer. His current research areas are fault detection and diagnosis and systems reliability.

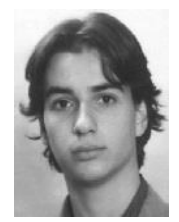

Antonio Correcher Salvador received a B.Sc. degree in automatic control and electronic engineering from the Polytechnic University of Valencia in 2001. He is currently working towards the Ph.D. degree in the Department of Systems Engineering at the Polytechnic University of Valencia. He is an assistant lecturer at this university. His research interest include discrete event systems, hybrid systems, and failure diagnosis.

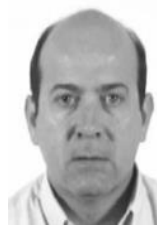

Francisco Morant received an M.Sc in Electrical Engineering from the Polytechnic University of Valencia in 1982, and a Ph.D. from the same university in 1985. In 1985 he joined the Systems and Control Engineering Department of the Technical University of Valencia, where he is an associate lecturer. His current research areas are expert systems control and fault detection and diagnosis. 